\title{
Simulation of transonic buffet with an automated zonal DES approach
}

\author{
Maximilian Ehrle ${ }^{1}$ (D) Andreas Waldmann ${ }^{1} \cdot$ Thorsten Lutz $^{1} \cdot$ Ewald Krämer $^{1}$
}

Received: 14 January 2020 / Revised: 8 July 2020 / Accepted: 19 August 2020 / Published online: 1 September 2020

(c) The Author(s) 2020

\begin{abstract}
A study of transonic buffet on the NASA Common Research Model at flight Reynolds numbers is presented. The ability of two different hybrid RANS/LES models as well as the URANS approach for resolving three-dimensional buffet motion was evaluated by means of spectral analysis. Automated Zonal DES and URANS simulations show similar results in terms of buffet frequency and spanwise propagation of buffet cells, whereas the Delayed Detached Eddy Simulation results indicate a strong interaction between flow separation and shock motion. The extracted characteristic frequencies which are associated with transonic buffet are located in a range of $S r=0.2-0.65$ for URANS and AZDES and are therefore in accordance with findings from related recent research. Furthermore, the simulation time series were investigated and a structure of spanwise moving buffet cells with varying convection speed and wavelength could be observed.
\end{abstract}

Keywords Transonic buffet · Aircraft aerodynamics · Hybrid RANS/LES · NASA Common Research Model

\section{Introduction}

Safety and efficiency improvements play a major role in the development of modern transport aircraft. Since cruise flight in the transonic regime is the primary part of a typical flight connection, the investigation of the aerodynamics in this phase is of special interest. This requires a better understanding of the flow effects determining its boundaries. The accurate prediction of aerodynamic phenomena that occur under these conditions remains a challenging task due to the occurrence of locally supersonic flow above the upper surface of the wing and the associated shocks and shock-boundary layer interaction. If the Mach number or the angle of attack are increased, these phenomena can result in transonic buffet, a complex unsteady shock motion coupled

Maximilian Ehrle

ehrle@iag.uni-stuttgart.de

Andreas Waldmann

waldmann@iag.uni-stuttgart.de

Thorsten Lutz

lutz@iag.uni-stuttgart.de

Ewald Krämer

kraemer@iag.uni-stuttgart.de

1 University of Stuttgart, Faculty 6: Aerospace Engineering and Geodesy, Institute of Aerodynamics and Gas Dynamics, Pfaffenwaldring 21, 70569 Stuttgart, Germany with shock-induced flow separation. Transonic buffet can lead to dynamic loads on the airplane structure, which causes a demand of improving its prediction. Furthermore, shock-induced flow separation results in an unsteady turbulent wake, which can interact with the aircraft's tailplane and induces fluctuating loads [12]. Because of the complex turbulent motion in the wake, scale resolving simulation methods are necessary to ensure a proper reproduction of flow physics in this area [19]. In addition, the knowledge of the influence of these conditions on the location of flow separation is essential for the prediction of the turbulent wake's propagation and development. As a consequence, it is imperative to validate the prediction of the phenomena in the vicinity of the wing surface with these methods, before moving on to studies on the flow physics and interactions in the wake.

\subsection{Studies and physics of the buffet phenomenon}

While transonic buffet on airfoils has been a well researched phenomenon in the last decades, giving rise to different approaches for its underlying physical mechanisms, e.g. [3, 17], an increasing number of studies on swept wings has been carried out in recent years to investigate buffet on more realistic configurations. These studies led to the conclusion that transonic buffet on three-dimensional configurations represents an even more complex phenomenon due to interaction 
with three-dimensional flow separation and spanwise flow effects. Iovnovich and Raveh [13] investigated the influence of sweep angle on the buffet phenomenon for generic wings by means of CFD. Their work comprises wings of finite and infinite aspect ratio. The latter were modeled with a zerothorder extrapolation boundary condition. They observed that the propagation of pressure disturbances in spanwise direction becomes dominant with increasing sweep angle. These pressure disturbances occur at the rear part of the shock in the area of the wing root and propagate toward the wing tip. The resulting shock-boundary layer-interaction leads to local shock oscillations, which move to the wing tip as socalled buffet-cells. This mechanism reveals higher buffet frequencies in comparison to two-dimensional buffet, which are attributed to an increased cross-flow with increasing sweep angle. However, the origin of the pressure fluctuations in the area of the wing root remains open. Another buffet model was proposed by Crouch et al. [4], who performed a global stability analysis based on URANS simulations of swept and unswept wings. They postulate that buffet on swept wings is associated with a global flow instability, but with a different primary instability mode than on two-dimensional or unswept wings, which agrees with the observed higher characteristic frequencies. For high-speed stall on airplane configurations, experimental studies were published for the NASA Common Research Model (CRM) [33] at low Reynolds numbers [15, 29] and at the AVERT model [5], which characterize the three-dimensional buffet phenomenon and describe the spanwise propagation of the observed buffet cells. Koike et al. [15] have established a classification of the shock oscillation on the CRM for three different angles of attack. This and earlier studies suggest that in contrast to the two-dimensional phenomenon at airfoils, transonic buffet at wings is accompanied by a broadband pressure fluctuation. Paladini et al. [21] evaluated various experimental investigations on 3D buffet and derived case-independent characteristic Strouhal numbers and spanwise convection velocities. This study also remarks that, in contrast to $2 \mathrm{D}$ buffet, fundamentally different physical mechanisms prevail and that the characteristic frequency ranges are wider than in the 2D case. Timme [31] investigated the buffet instability mechanisms at the CRM by means of global modal analysis of RANS simulations. He concluded that buffet onset is connected with one single unstable oscillatory eigenmode. Moreover, additional unstable oscillatory modes beyond buffet onset agree well with the frequency range of transonic buffet, which is observed in recent research. A recent review of investigations on transonic buffet by Giannelis et al. [9] concluded that a general model for the description of transonic buffet has not yet been attained.

While the previous studies focus on the simulation of transonic buffet by means of URANS approaches, Sartor et al. [23] applied the hybrid RANS/LES model Delayed
Detached Eddy Simulation (DDES) for buffet conditions on an airplane configuration. The simulation results showed a good agreement between the statistics of the surface pressure distribution and the underlying experiments. They concluded that the unsteady RANS approach is able to reproduce the major flow phenomena associated with transonic buffet. In addition, the DDES method showed advantages regarding the reproduction of flow physics and agreement with experiments. Ishida et al. [14] were also able to qualitatively reproduce transonic buffet at the CRM using a Zonal Detached Eddy Simulation (ZDES) method at low Reynolds numbers, whereas no quantitative agreement with the measured data could be achieved. Hashimoto et al. [10] improved these results by means of an additional wall model and were able to observe 3D buffet.

Several studies on the simulation setup for the investigation of transonic buffet on the CRM at flight Reynolds numbers have been made in recent years. Illi et al. [12] identified two principal issues of linear eddy viscosity based URANS simulations: an excessive, unphysical corner separation in the wing-body junction affecting the buffet motion and the excessive dissipation of turbulent structures in the separated wake of the CRM. As Togiti et al. [32] showed, the corner flow separation can be prevented using Reynolds stress transport models. They also observed a significant reduction of the corner flow separation by applying eddy viscosity models in combination with the quadratic constitutive relation (QCR) [25], which improves the prediction of Reynolds stress anisotropy. The wake dissipation issue can be tackled with hybrid RANS/LES models that allows further investigation of the turbulent wake downstream from areas with shock-induced flow separation. For this reason Lutz et al. [19] employed a first version of the hybrid RANS/LES model Automated Zonal DES (AZDES) coupled to the SSG/ LRR- $\omega$ Reynolds stress model to simulate the shock-induced separated wake behind the CRM and achieved encouraging results. The present study focuses on the capability of different simulation approaches to reproduce three-dimensional transonic buffet. The first sections of the paper introduce and compare the applied hybrid RANS/LES models and present the numerical setup. Subsequently, spectral analyses of the pressure coefficient on the wing surface are discussed. In the last part, a study of the spanwise propagation and properties of the observed buffet cells is presented.

\section{Hybrid RANS/LES-modeling}

While the main buffet phenomena can be captured by URANS, as Crouch et al. [4], Thiery and Coustols [30] or Sartor et al. [23] demonstrated, these methods are unsuitable for the further investigation of the phenomena in the turbulent wake due to excessive dissipation of turbulent motion. 
On the other hand, the computational cost of fully resolved or wall resolved LES for an aircraft configuration at flight Reynolds numbers is too high, even for current supercomputers. However, the conditions on the separation location of the turbulent wake as well as a proper reproduction of wake turbulence are both essential to successfully perform simulations and investigations on the entire airplane. Hence, hybrid RANS/LES models such as the Detached Eddy Simulation (DES) are an appropriate way to tackle this problem. These methods combine low computational cost in the vicinity of the wing surface (RANS mode) with scale resolving behavior in regions of separated flow and turbulent motion (LES mode). The popularity and use of such methods has led to numerous modifications of the original DES model to mitigate the dependency of RANS/LES areas on mesh resolution. The Delayed Detached Eddy simulation (DDES) [27], for example, considers local flow quantities to shield the boundary layer so that LES mode is only activated in areas of detached flow and thus reduces problems such as grid-induced flow separation (GIS). This function is implemented by introducing a delay parameter $f_{\mathrm{d}}$ in the length scale formulation

$L_{\mathrm{DDES}}=L_{\mathrm{RANS}}-f_{\mathrm{d}} \cdot \max \left(0, L_{\mathrm{RANS}}-C_{\mathrm{DES}} \Delta\right)$,

where $C_{\mathrm{DES}}$ is a constant of order 1 and $\Delta$ is the chosen filter width model. Hybrid RANS/LES models have been used in the high-speed stall of transport aircraft configurations for low Reynolds numbers [14, 23]. Furthermore, their application was successfully tested on the CRM in low-speed stall conditions at high angles of attack [34]. Despite the mentioned improvements, grid-induced flow separation is still an issue that concerns smooth geometries without a clearly defined separation point. Too fine grid resolution may cause a collapse of the shielding in the boundary layer area and lead to an incursion of the LES region into parts of the boundary layer as well as to premature flow separation [1]. Schulte am Hülse [11] showed that DDES is capable of resolving the separated wake of the OAT15A airfoil. However, he pointed out that shock motion and position are strongly influenced by the hybrid model, the grid resolution and other computational parameters. Grids with a too fine resolution cause the shock to remain in a far upstream position. Consequently, massive separation occurs and buffet cannot be reproduced. These observations are in agreement with those of Garnier and Deck [8], who described a connection of the periodic shock motion behavior with the LES filter width.

\subsection{Automated zonal DES (AZDES) approach}

As a consequence of the issues of conventional hybrid RANS/LES approaches described in the previous section, the AZDES method was proposed by Schulte am Hülse [11] and first prototype applications were shown at the CRM [19]. The aim of this method is to extend the shielding of the attached boundary layer and consequently treat the shock and boundary layer region in RANS mode. The resolution of separated flow and the turbulent wake in LES mode enables further investigations of the flow phenomena in these regions. This scale resolving behavior is realized by activating the DDES model outside of the pre-defined RANS areas. The main difference to Deck's [6] ZDES model is the zone derivation based on an evaluation of solution-based quantities of a precursor URANS simulation. Consequently, regions of separated flow can be treated in DDES mode independently of the zonal DES parameters that relate to the wall distance. The extend of this area can be influenced by the user. Similar to ZDES, a blending function $f_{\mathrm{a}}$ separates the zones of RANS behavior, where $f_{\mathrm{a}}=0$, and DDES behavior, where $f_{\mathrm{a}}=1$. Based on two user-defined, wall distance dependent parameters $d_{\text {RANS }}$ and $d_{\text {DES }}$ as well as on areas of separated flow, the RANS/LES interface is determined in three steps. In order to enable early switching from RANS mode to DDES mode in areas of separated flow, $f_{\mathrm{a}}$ is initially defined by a user-defined threshold value $L_{\mathrm{c}}$ of the turbulent RANS length scale $L_{\mathrm{t}}$. The distribution of $L_{\mathrm{t}}$ in the flow field is cumulated in a precursor URANS simulation of the same flow conditions. This intends to introduce an estimate of the overall extent of the separation region into the zone derivation and influences $f_{\mathrm{a}}$ as described in Eq. 2.

$f_{\mathrm{a}}^{L_{\mathrm{c}}}=\frac{1}{2} \tanh \left[8 \cdot\left(\frac{L_{\mathrm{t}}}{L_{\mathrm{c}}}-1\right)\right]+\frac{1}{2}$

Subsequently, $f_{\mathrm{a}}^{L_{\mathrm{c}}}$ can be modified by $d_{\text {RANS }}$. Close to the wall, i.e. when the wall distance $d<d_{\text {RANS }}$, the value of $f_{\mathrm{a}}$ follows

$f_{\mathrm{a}}^{d_{\mathrm{RANS}}}=\frac{1}{2} \tanh \left[8 \cdot\left(\frac{d}{d_{\mathrm{RANS}}}-1\right)\right]+\frac{1}{2}$, for $0<d<d_{\mathrm{RANS}}$.

Finally, the parameter $d_{\mathrm{DES}}$ defines a wall distance, above which resolved content is allowed independently of the state of flow separation. This parameter modifies $f_{\mathrm{a}}^{L_{\mathrm{c}}}$ where $d>d_{\mathrm{DES}}$ with

$f_{\mathrm{a}}^{d_{\mathrm{DES}}}=\frac{1}{2} \tanh \left[8 \cdot\left(\frac{d}{d_{\mathrm{DES}}}-1\right)\right]+\frac{1}{2}$, for $d_{\mathrm{DES}}<d<\infty$.

A schematic representation of all parameters is illustrated in Fig. 1. Areas with $f_{\mathrm{a}}=0$ (red) are treated in RANS mode whereas $f_{\mathrm{a}}=1$ (white) allows LES mode by activating the DDES model. Based on parametric studies at the OAT15A airfoil and the CRM, values of $d_{\text {RANS }} / \mathrm{MAC}=0 \%$ and $d_{\mathrm{DES}} / \mathrm{MAC}=6 \%$ are considered to be case-independent and 


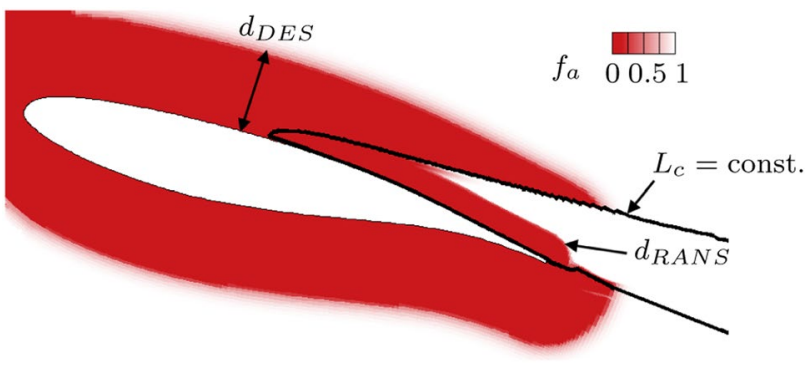

Fig. 1 Sketch of $f_{\mathrm{a}}$ distribution around an airfoil and the relevant AZDES parameters

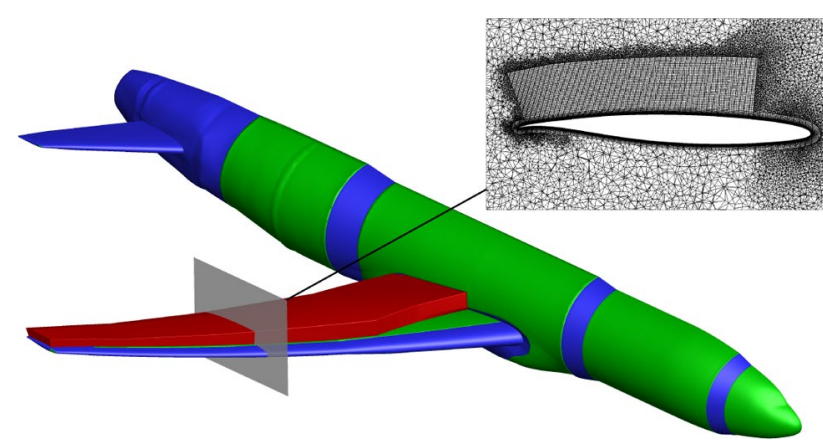

Fig. 2 Outer layer of the CRM boundary layer mesh, hexahedra: blue, prisms: green, hexahedral mesh on the wing surface: red, slice through the wing mesh at $\eta=0.6$

independent of the turbulence model. The value of $L_{\mathrm{c}} / \mathrm{MAC}$ is selected so that the DDES area has only a minor influence on the buffet behaviour determined by the URANS mode. However, since the buffet characteristics are sensitive to this parameter, it should be verified for new flow cases and turbulence models. It is assumed that $L_{\mathrm{c}} / \mathrm{MAC}=7 \%$, which is used in this work, can be used as a reference value for simulations of aircraft configurations with the SA turbulence model. For a more reliable statement towards the generality of all three parameters the testing of the AZDES model on other aircraft configurations is part of future research.

\section{Computational setup}

In the context of the European ESWI ${ }^{\mathrm{RP}}$ project, the flow around the CRM under transonic conditions was measured in a wind tunnel campaign at the European Transonic Wind Tunnel (ETW) in Cologne, Germany [18]. The simulations in the present work replicate this test campaign's inflow conditions to ensure comparability and enable future validation of simulation data. However, the wind tunnel support system is not considered. The results in the following section were obtained using a hybrid grid that represents a half model of the CRM. The wind tunnel model's scale of $2.7 \%$ leads to a
Table 1 Flow conditions and geometric parameters

\begin{tabular}{llllll}
\hline$M a_{\infty}$ & $R e_{\infty}$ & $p_{0}$ & $T_{0}$ & MAC & $\alpha$ \\
\hline 0.85 & $30 \times 10^{6}$ & $303 \mathrm{kPa}$ & $115 \mathrm{~K}$ & $0.189144 \mathrm{~m}$ & $5^{\circ}$ \\
\hline
\end{tabular}

Table 2 Gas constant and sutherland parameters for nitrogen

\begin{tabular}{llll}
\hline$R$ & $C$ & $T_{0}$ & $\mu_{0}$ \\
\hline $296.8 \frac{\mathrm{J}}{\mathrm{kg} \mathrm{K}}$ & $111.0 \mathrm{~K}$ & $300.55 \mathrm{~K}$ & $17.81 \times 10^{-6}$ \\
\hline
\end{tabular}

mean aerodynamic chord (MAC) of $0.189 \mathrm{~m}$. The grid, as shown in Fig. 2, consists of near-wall prisms and hexahedral elements with a proper boundary layer resolution and $y^{+}<1$ on the fuselage and wing surfaces. The majority of the upper surface of the wing is discretized using quadrilateral elements, which are kept as isotropic as practically possible. Additionally, a block of near-isotropic hexahedra above the boundary layer mesh of the wing's suction side is embedded in the grid. The cell size of this block was chosen according to a local convective CFL (Courant-Friedrichs-Levy) number of 1 following the guidelines of [28]. This ensures a sufficient spatial resolution of the shock and the separated flow area for the use of hybrid RANS/LES models. During the wind tunnel campaign, the aeroelastic deformation of the CRM geometry was measured using stereo pattern tracking (SPT). For the present flow conditions, a maximum twist of $2^{\circ}$ and an upward bending of $30 \mathrm{~mm}$ were observed near the wingtip. This results in a change of the local angle of attack. Therefore, the impact of these deformations on the local pressure distribution, the shock position and transonic buffet is expected to be significant. Consequently, the measurement data of the wing's twist and bending distribution was incorporated in the CFD geometry by means of a static mesh deformation. A more thorough description of the deformation mechanism is provided in [19]. The farfield of the grid with a radius of 50 times the model length is filled up with tetrahedra, which leads to an overall number of grid points of around $36 \times 10^{6}$.

Table 1 lists the flow conditions of the simulations. Due to the cryogenic conditions, nitrogen with the sutherland parameters listed in Table 2 is used as working fluid. According to Schulte am Hülse [11], Timme [31] and Sugioka et al. [29] transonic buffet can be expected for these values.

All simulations in this paper were performed using the TAU flow solver by DLR [24]. TAU is a finite volume solver using unstructured grids via a cell-vertex dual grid approach. Second-order accuracy in space is achieved by a central differencing scheme for the convective terms. TAU's matrix type artificial dissipation is chosen to reduce the numerical dissipation of small scale structures. More specifically, a 
ratio between matrix and scalar dissipation of $80 / 20$ and a fourth-order dissipation coefficient of 1/256 were chosen. This represents the dissipation settings that come closest to the recommendation for hybrid RANS/LES simulations in the DLR TAU-code user guide [7] and still allow a numerically stable solution for all simulations. The skew symmetric scheme proposed by Kok [16] and a first-order Roe scheme are applied for the discretization of the meanflow fluxes and the turbulence fluxes, respectively. Time integration is realized with an implicit backward Euler scheme. A lower-upper symmetric Gauss-Seidel scheme is used as a linear solver. The dual time-stepping scheme is applied for all unsteady simulations and enables second-order accuracy in time. The physical time step size of $6.67 \times 10^{-6} \mathrm{~s}$ represents 150 time steps per convective time unit $t_{\infty}=\mathrm{MAC} / u_{\infty}$, the number of inner iterations was set to 100 . A CFL number of the implicit pseudo time steps of two is chosen. Acceleration of convergence was realized with a $3 \mathrm{v}$ multigrid cycle. Two turbulence models were employed: the Spalart-Allmaras (SA) model [26], specifically the version without trip-term $f_{\mathrm{t}}$ and turbulence suppression term $f_{\mathrm{t} 2}$, and the SSG/LRR- $\omega$ Reynolds stress model [2]. The QCR extension [25] was activated for the SA model. Initial steady-state computations with a ramp-up of the angle of attack until $\alpha=5^{\circ}$ were performed to gain a proper starting point for the unsteady simulations. The AZDES mode requires a precursor URANS simulation, which was run over 80 convective times related to the MAC in order to get a representative distribution of the turbulent length scale. The $\Delta_{\max }$ filter, based on the maximum edge length of the grid cells, is used for the hybrid RANS/LES simulations.

\section{Results and discussion}

In the following section, the results of URANS, AZDES and DDES simulations will be compared to assess the different models' capabilities of resolving the shock motion on the wing's suction side. As already indicated in the previous section, a study of buffet onset criteria at the CRM [11] suggested that, at the underlying inflow conditions, buffet sets in at $\alpha>3.7^{\circ}$. $\mathbf{R 2}: \mathbf{S 1}$ Furthermore, investigations at lower Reynolds numbers of Timme [31] and Sugioka [29] also predict buffet onset in the range of $\alpha \approx 3.7^{\circ}$. These studies comprise a range of Reynolds numbers from $1.5 \times 10^{6}$ to $30 \times 10^{6}$. A fully turbulent boundary layer is assumed in all studies. This indicates a minor influence of the Reynolds number on the onset of transonic buffet under the condition of a turbulent boundary layer in the shock region. Therefore, it is expected that buffet motion occurs at the current flow conditions and the investigated angle of attack. An evaluation of the turbulence models shows that simulations with the SSG/LRR- $\omega$-Reynolds stress model for inflow conditions according to Table 1 lead to a static shock position without buffet motion. The SA turbulence model with QCR-extension developed an unsteady motion of spanwise convecting buffet cells. The different behavior with regard to buffet motion can be attributed to different levels of eddy viscosity. The SA model yields significantly lower values of eddy viscosity near the wing surface and in the region of separated flow compared to the RSM. This may lead to a damping of the shock oscillation in case of the latter, which finally leads to a static shock position. This dependency of the ability to resolve transonic buffet on the eddy viscosity level was also observed by Sartor et al. [23]. The differences in the shock unsteadiness between the SA model and the SSG/LRR-RSM can be seen in Fig. 3 by means of the root mean square of the surface pressure coefficient, shown on the wing's suction side. Due to these findings, the present work focuses on simulations with the SA turbulence model to capture the unsteady phenomena.

\subsection{Comparison of hybrid RANS/LES models}

To obtain a better understanding of the properties of the twohybrid RANS/LES approaches concerning 3D buffet, we analyze the distribution of RANS/LES zones on the CRM wing. As described in Sect. 2.1, it is the distribution of the turbulent length scale from a URANS simulation, combined with three user-adjustable parameters, that determines the AZDES model's distribution of zones with RANS and DES behavior. In contrast to DDES, this interface stays constant over the simulation time and does not change depending on the local flow conditions. Figure 4 visualizes the turbulent RANS length scale $L_{\mathrm{t}}$ in a slice at $\eta=0.6$. The chosen threshold $L_{\mathrm{c}} / \mathrm{MAC}=7 \%$, which is used for the AZDES zone interface definition in this work, is plotted in blue.

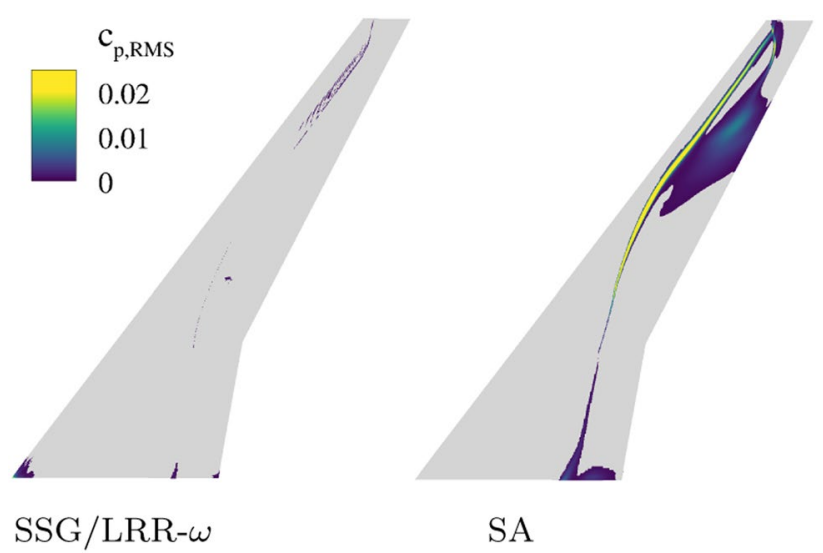

Fig. 3 RMS of the pressure coefficient, URANS simulations with the SSG/LRR- $\omega$-RSM and the SA turbulence model, blanking of areas $c_{\mathrm{p}, \mathrm{RMS}}<5 \times 10^{-4}$ 


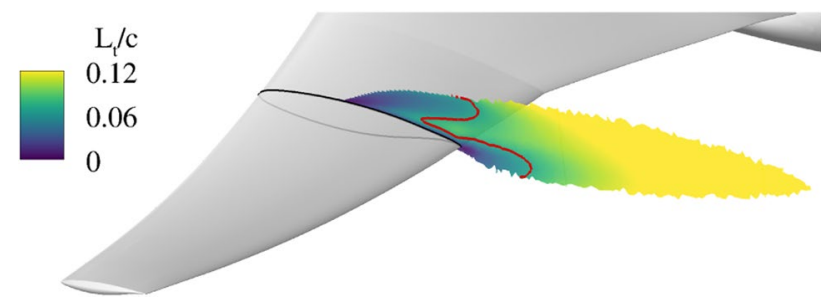

Fig. 4 Distribution of the turbulent length scale on the CRM wing at $\eta=0.6$ and AZDES cutoff value $L_{\mathrm{c}} / \mathrm{MAC}=7 \%$ in red

Based on this value, Fig. 5a shows the RANS zone distribution in several slices at the CRM wing. A hybrid mode value of 0 represents RANS behavior, a value of 1 DES behavior of the hybrid model. Time-averaged surface pressure contours are shown as a reference. In comparison, Fig. $5 b$ shows the respective RANS area distribution of the DDES simulation. It can be seen that the AZDES approach and the chosen settings lead to a thicker RANS area around the wing surface, shielding the attached boundary layer from resolved turbulence. At the same time, the LES region approaches the wing surface in areas of separated flow, which is detected via an increased value of $L_{\mathrm{t}}$, see Fig. 4.

In this flow case, the area of flow separation is located approximately between $\eta=0.4$ and $\eta=0.7$. Due to these observations, AZDES is expected to have a RANS like behavior in the vicinity of the wing, with only minor interaction between the resolved turbulence and the shock. In contrast to this, DDES leads to a thin RANS layer, particularly after the shock, which suggests a stronger impact of resolved LES content on the boundary layer and the shock. Figure 6 shows a slice of the mean pressure coefficient at $\eta=0.603$ of URANS, AZDES and DDES simulations, as well as experimental data from ETW wind tunnel tests of the $\mathrm{ESWI}^{\mathrm{RP}}$ project [18]. All three computations show reasonable agreement with the experiment in the shock region. However, an accurate validation of the shock position is difficult due to the coarse spacing of pressure taps in this area. Out of all three approaches, DDES yields the most upstream and URANS the most downstream shock

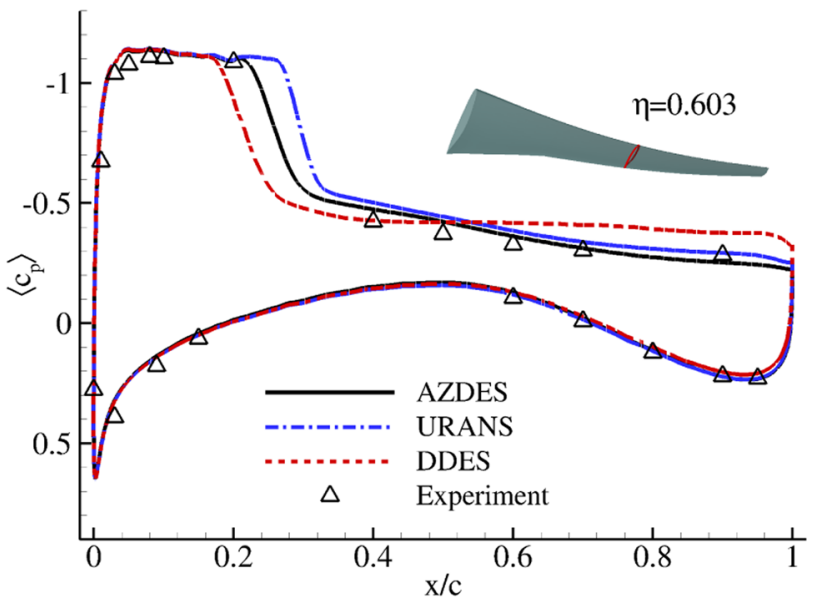

Fig. 6 Mean pressure coefficient at a slice of $\eta=0.603$, URANS, DDES, AZDES, Experimental data from the ESWI ${ }^{\mathrm{RP}}$ project [18] $M=0.85, \operatorname{Re}=30 \times 10^{6}, \alpha=5^{\circ}$

position. AZDES and URANS predict the mean surface pressure in all parts of this slice in good accordance with the wind tunnel results, whereas DDES underestimates the pressure level and its chordwise gradient in the rear part of the wing's suction side.

\subsection{Spectral analysis results}

Since no unsteady experimental data was gathered during the $\mathrm{ESWI}^{\mathrm{RP}}$ measurement campaign, unsteady measurements of Koike et al. [15] are consulted. These data were gathered at an $80 \%$ scale CRM wing, a slightly lower angle of attack of $4.84^{\circ}$ and a lower Reynolds number of $R e=1.515 \times 10^{6}$. Since the Reynolds number differs by a factor of 20 and there is a discrepancy in the angle of attack, the measurement data can only be used to enable an approximate, qualitative comparison of unsteady phenomena. Figure 7 shows the root mean square values of the pressure coefficient on a slice through the wing at $\eta=0.6$. This slice corresponds to the position investigated by Ohmichi et al. [20] as well as one spanwise position of the unsteady pressure measurements by Koike et al. [15]. The experimental data was
Fig. 5 Mean pressure coefficient and slices representing the RANS zone distribution (red) of AZDES and DDES on the wing suction side, $\mathrm{SA}+\mathrm{QCR}$, $M=0.85, R e=30 \times 10^{6}, \alpha=5^{\circ}$

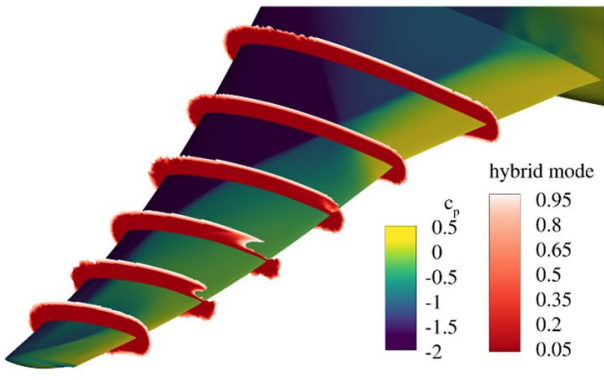

(a) AZDES

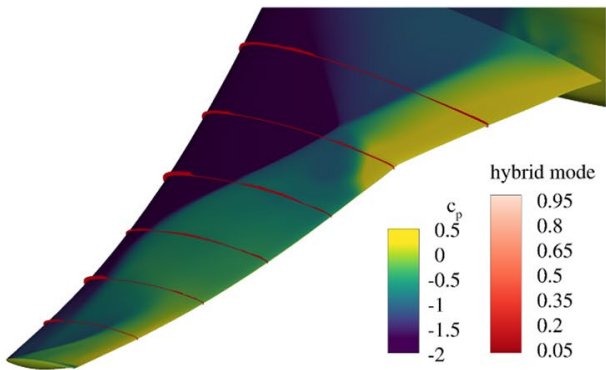

(b) DDES 


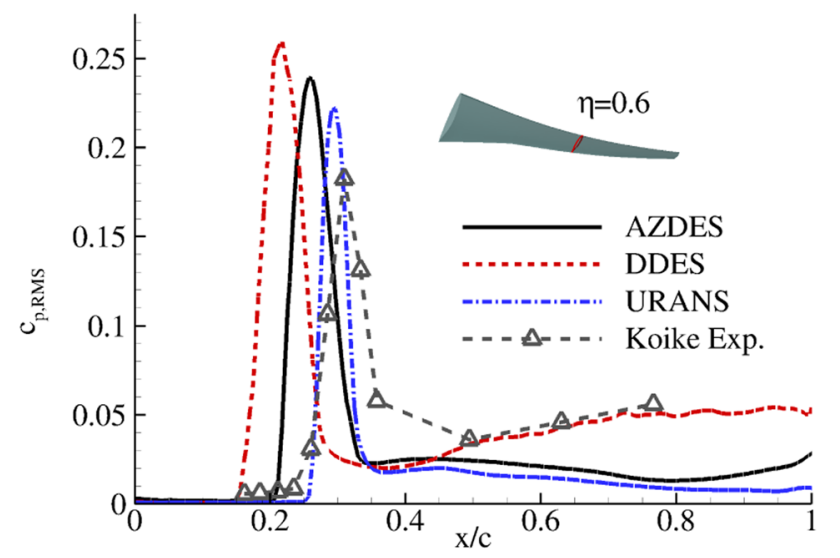

Fig. 7 RMS of the pressure coefficient at a slice of $\eta=0.6$ on the wing's suction side, $M=0.85, R e=30 \times 10^{6}, \alpha=5^{\circ}$, experimental data from Koike et al. [15]

included as "Koike Exp." in the figure. URANS shows the most downstream position of the peak of pressure fluctuations at $x / c=0.3$, whereas AZDES and DDES lead to an upstream shift to $x / c=0.26$ and $x / c=0.22$, respectively. The magnitude of the pressure fluctuations increases from URANS over AZDES to DDES. All three simulations yield a higher level of pressure fluctuations than the experiment. DDES leads to an overall higher fluctuation level in the rear half of the wing, whereas AZDES shows only a small increase in pressure fluctuations near the trailing edge in comparison to URANS. These results suggest a varying influence of resolved turbulence on the wing surface. However, DDES lies closest to the experimental data of Koike et al. [15] in the rear half of the wing. In contrast, the fluctuation peak of the URANS lies closest to the experimental data, which reveal the maximum pressure fluctuations at $x / c=0.31$. Koike et al. observed a downstream shift of the shock position and an increase in pressure fluctuations with increasing Reynolds number. Therefore, the higher RMS values of $c_{\mathrm{p}}$ in the simulations of this study can be considered reasonable. These findings lead also to the conclusion that the farthest downstream shock position predicted by URANS is closest to reality. All three simulations predict the peak pressure fluctuation further downstream than Ohmichi et al.'s ZDES results [20]. Overall, AZDES shows results that are closer to URANS, as it was expected by investigating the RANS zone distribution.

In the following, three points at this spanwise position of the wing are investigated in more detail. The first point at $x / c=\max \left(c_{\mathrm{p}, \mathrm{RMS}}\right)$ represents the maximum of the pressure fluctuations for each simulation approach. Point 2 and 3 correspond to $x / c=0.36$ and $x / c=0.77$, which were investigated by Koike et al. [15] and Ohmichi et al. [20], respectively. To identify characteristic Strouhal numbers that correspond to buffet motion and other unsteady phenomena

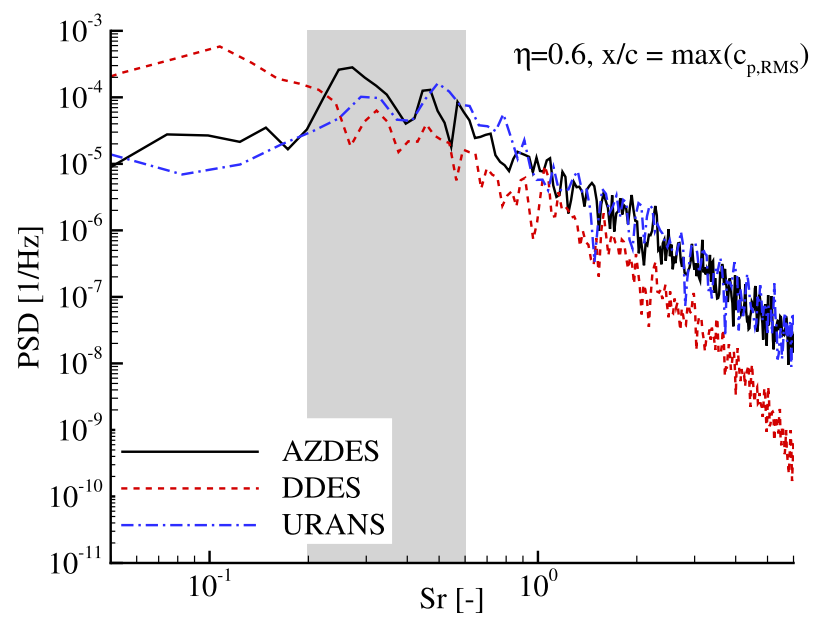

Fig. 8 Spectra of the pressure coefficient obtained with the Welch method, $\eta=0.6, x / c=\max \left(c_{\mathrm{p}, \mathrm{RMS}}\right), M=0.85, \mathrm{Re}=30 \times 10^{6}, \alpha=5^{\circ}$. The $\mathrm{Sr}$ range attributed to transonic buffet in recent research is highlighted in grey

on the wing's suction side, we analyze a spectral analysis of the pressure fluctuations.

The time interval includes 15000 physical timesteps of the computation representing 100 convective times, whereas the surface solution was sampled at every tenth timestep for the spectral analysis. The power spectral density (PSD) of the pressure coefficient was calculated with Welch's method and divided into two segments to obtain accurate spectra in the relevant frequency range. The available spectral resolution is limited by the number of samples per segment to $\Delta S r=0.022$ which does not allow statistically converged low-frequency analysis below a value of approximately $S r=0.1$. Results in that frequency range can still indicate trends but have to be verified in future work. Since the buffet phenomenon's characteristic frequency range is expected to be above this value, the resolution is satisfying for the analyses carried out in the present paper. For future investigations of lowfrequency effects, a longer time series will be required, which significantly increases the computational effort. The Strouhal range of $0.2-0.6$ in which recent literature locates three dimensional transonic buffet is highlighted in grey in the following figures.

Figure 8 shows the PSD of the pressure coefficient at the maxima of $c_{\mathrm{p}}$ fluctuations for URANS, AZDES and DDES simulations. These points (Point 1 ) were chosen to directly compare the three computational approaches in an area where shock oscillations can be expected in all three cases. DDES reveals a higher level of PSD in the range of $\mathrm{Sr}=$ $0.1-0.12$. This result is statistically not converged but hints at a peak level in that area. 


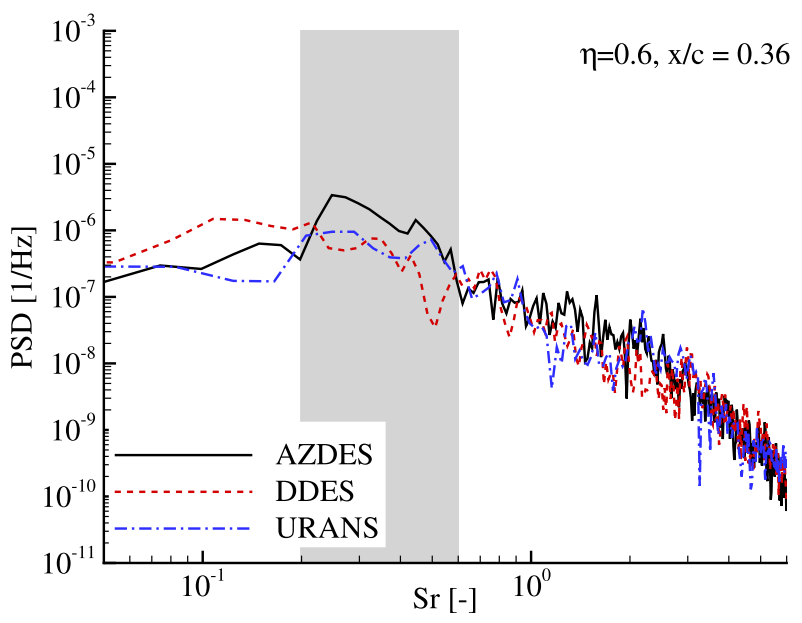

Fig. 9 Spectra of the pressure coefficient obtained with the Welch method, $\eta=0.6, x / c=0.36, M=0.85, R e=30 \times 10^{6}, \alpha=5^{\circ}$. The $\mathrm{Sr}$ range attributed to transonic buffet in recent research is highlighted in grey

No distinctive other frequencies can be observed for this simulation. AZDES shows a broadband elevation of PSD level in between $S r=0.2$ and 0.55 . URANS yields $S r=$ $0.25-0.65$ as a dominant frequency range. Overall, AZDES and URANS show a similar distribution of frequencies that agree well with the highlighted range, whereas DDES predicts lower peak frequencies.

Figure 9 illustrates the surface pressure spectrum at a chordwise position of $x / c=0.36$ (Point 2). DDES yields a dominant frequency range of $S r=0.09-0.4$, AZDES and URANS show high PSD levels for $S r=0.2-0.55$. Overall, the spectra in Figs. 8 and 9 show the most significant pressure fluctuations in URANS and AZDES to be in the same frequency range as described in recent research [20]. DDES peaks in a lower frequency range as the other two approaches.

The surface pressure spectra of the three simulations at $x / c=0.77$ (Point 3) that represents a point in the area of separated flow in Fig. 10 indicate the same tendency of a high fluctuation level at lower frequencies for DDES. This peak is located between $S r=0.094$ and $S r=0.15$ whereas AZDES and URANS yield peaks in the area of $S r=0.2$ and 0.33. DDES shows a higher fluctuation level across the entire frequency range, while AZDES and URANS predict a more significant spectral decay in the high frequency range. This behavior suggests the presence of resolved turbulence in the area of separated flow for DDES, whereas AZDES shows an URANS like behavior. Overall, the spectra in the area of separated flow show increased fluctuation levels in the same characteristic frequency range attributed to transonic buffet. This suggests, that buffet motion affects the development and characteristics of wake turbulence.

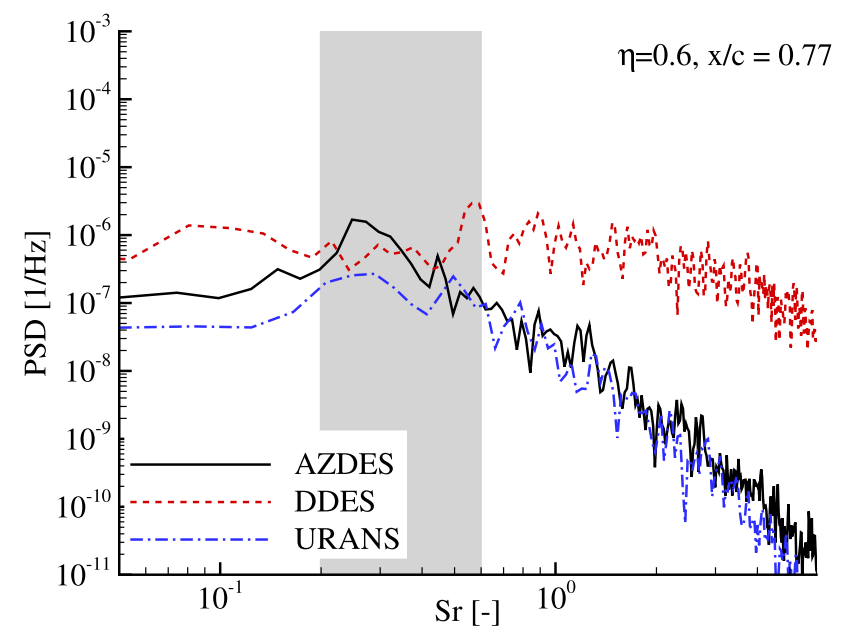

Fig. 10 Spectra of the pressure coefficient obtained with the Welch method, $\eta=0.6, x / c=0.77$, URANS, DDES, AZDES, $M=0.85, R e=30 \times 10^{6}, \alpha=5^{\circ}$. The $S r$ range attributed to transonic buffet in recent research is highlighted in grey

In addition to the mid-wing position at $\eta=0.6$, the wing's suction side in the shock area near the fuselage and at the outboard wing section were examined. As already indicated by the RMS value of the pressure coefficient in Fig. 3, only minor fluctuations can be observed in the inboard shock region and the shock in this area stays at a steady position. The investigation of the shock motion at $\eta=0.95$, which is located outboard of the separation zone near the wing tip, reveals a movement of the whole shock front parallel to the leading edge. Figure 11 shows the observed most upstream (red) and downstream (black) shock positions of the AZDES simulation in this area by means of a contour line representing $c_{\mathrm{p}}=-0.73$. Figure 12 illustrates the spectra of the surface pressure coefficient at $\eta=0.95$ and a local chord of $x / c=\max \left(c_{\mathrm{p}, \mathrm{RMS}}\right)$ (Point 4$)$. The spectra of URANS and AZDES reveal no elevated amplitudes in the range of $S r=0.3-0.6$ that could be connected to the motion of buffet cells as in the previously analyzed positions. Conversely, higher levels of the PSD at frequencies of $S r=0.2$ and below for the URANS and AZDES simulations hint at low-frequency fluctuations. This could be connected to the described shock motion in the outer part of the wing. DDES shows a peak between $S r=0.3$ and $S r=0.4$ but no regular shock movement can be observed in this area of the wing.

In comparison to URANS and AZDES, DDES shows a higher fluctuation level at frequencies in the range of $\mathrm{Sr}=$ 0.1-0.4. Furthermore, DDES shows higher levels of PSD for the whole frequency range up to high frequencies in the area of flow separation. Investigation of the time-resolved shock movement shows that DDES does not produce a periodic movement of buffet cells. Contrarily, a more chaotic, 
Fig. 11 Contours of instantaneous pressure coefficient for the AZDES simulation and most upstream (red) and downstream (black) shock position in the outer wing area $M=0.85, \operatorname{Re}=30 \times 10^{6}, \alpha=5^{\circ}$

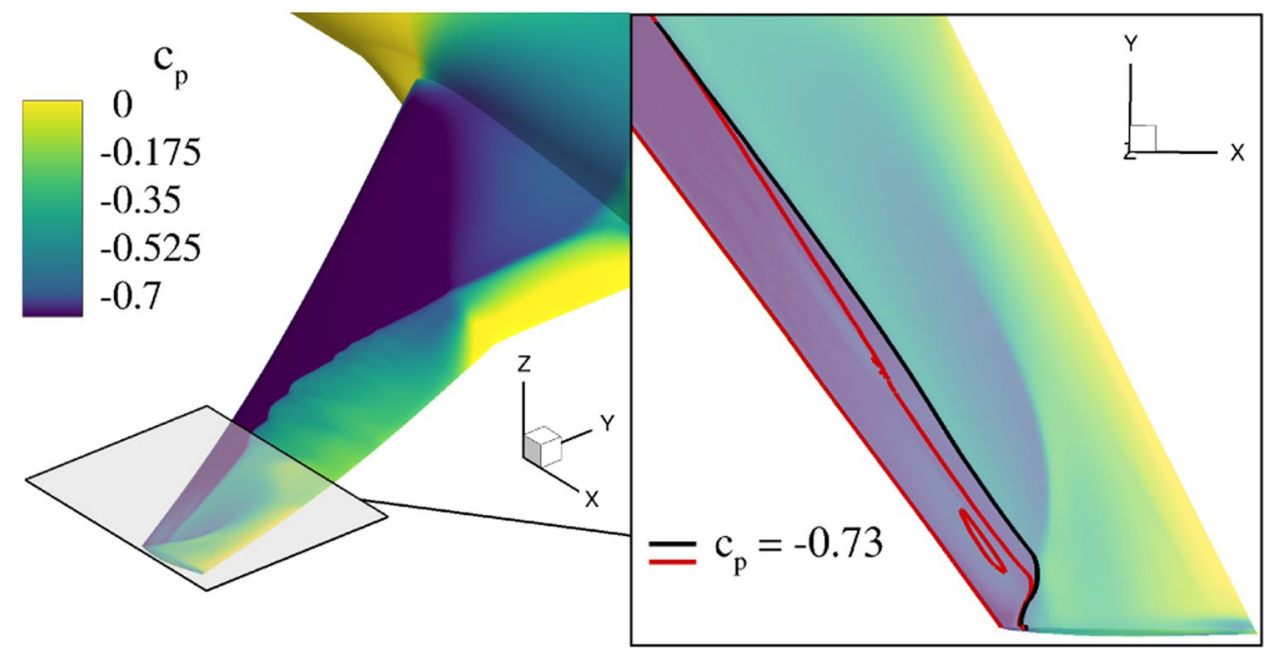

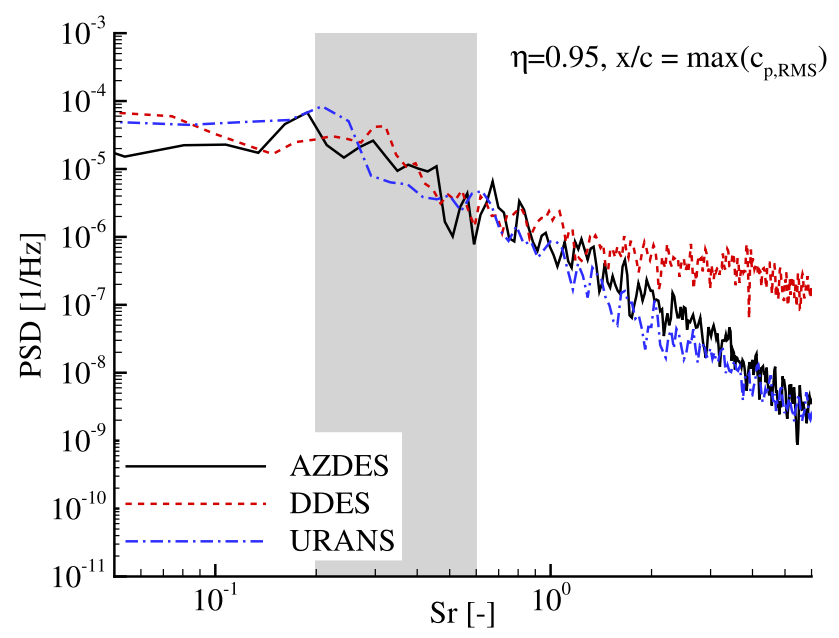

Fig. 12 Spectra of the pressure coefficient obtained with the Welch method, $\eta=0.95, x / c=\max \left(c_{\mathrm{p}, \mathrm{RMS}}\right)$, URANS, DDES, AZDES, $M=0.85, R e=30 \times 10^{6}, \alpha=5^{\circ}$. The $S r$ range attributed to transonic buffet in recent research is highlighted in grey

irregular movement of the shock can be observed. This behavior might be due to interactions between the separated flow and the shock, since a large portion of the separated flow region is treated in LES mode, as already demonstrated in Fig. 5b. On the contrary, AZDES and URANS predict a similar range of dominant Strouhal numbers $S r=0.2-0.65$ at the observed points at $\eta=0.6$.

Among others, Crouch et al. [4], Plante et al. [22] and Dandois et al. [5] describe a connection between the wing sweep and the dominant $\mathrm{Sr}$ number range of transonic buffet. They observed that the Strouhal number increases with increasing sweep angle. This effect must be considered when comparing the CRM with a sweep angle of $35^{\circ}$ to other wings and aircraft configurations. In agreement with those findings the range of $\mathrm{Sr}$ observed in this work is slightly above that of wings with smaller sweep angles. Sartor et al. [23] localized the buffet phenomenon for a wing body configuration with a sweep angle of $25^{\circ}$ in a frequency range of $150-300 \mathrm{~Hz}$, which corresponds to a $\mathrm{Sr}$ range of 0.16-0.32. Paladini et al. [21] connected transonic buffet on several wings with a sweep angle of $30^{\circ}$ with local pressure variations at $S r=0.2-0.3$. Despite this agreement, other geometric parameters such as taper and twist can also influence the buffet behaviour. The comparison with other investigations on the CRM allows a more specific insight into the influence of flow conditions on the buffet phenomenon. Ohmichi et al. [20] linked the three-dimensional transonic buffet on the CRM wing with Strouhal numbers $\mathrm{Sr}=$ 0.2-0.6. The experimental investigation of Koike et al. [15] revealed a shock wave oscillation in the region of $\mathrm{Sr}=0.3$. Furthermore, Timme [31] observed a band of eigenvalues with reduced decay rate at the CRM for $\mathrm{Sr} \approx 0.3-0.7$. Those results are consistent with the Strouhal numbers associated with buffet in this paper. The investigated Reynolds numbers of $1.5 \times 10^{-6}$ and $5 \times 10^{-6}$, respectively are in all three cases significantly lower than in the present case. Nevertheless, a similar frequency range for transonic buffet is obtained in all investigations. This suggests that the characteristic buffet Strouhal number range only shows minor dependency on the Reynolds number and a qualitative comparison of these publications with results of the present work is justifiable. The simulations in the present work do not reproduce the low-frequency peak of $S r=0.06$ in the pressure spectrum at $x / c=0.36$, which was detected by Ohmichi et al. [20]. This might also be due to an insufficient frequency resolution of the spectral analysis and the lack of statistically converged data in the low-frequency range. Consequently, no reliable comparison with these data in that frequency range is possible and has to be part of future work. A more detailed analysis of the spanwise moving buffet cells is subject of Sect. 4.3. 


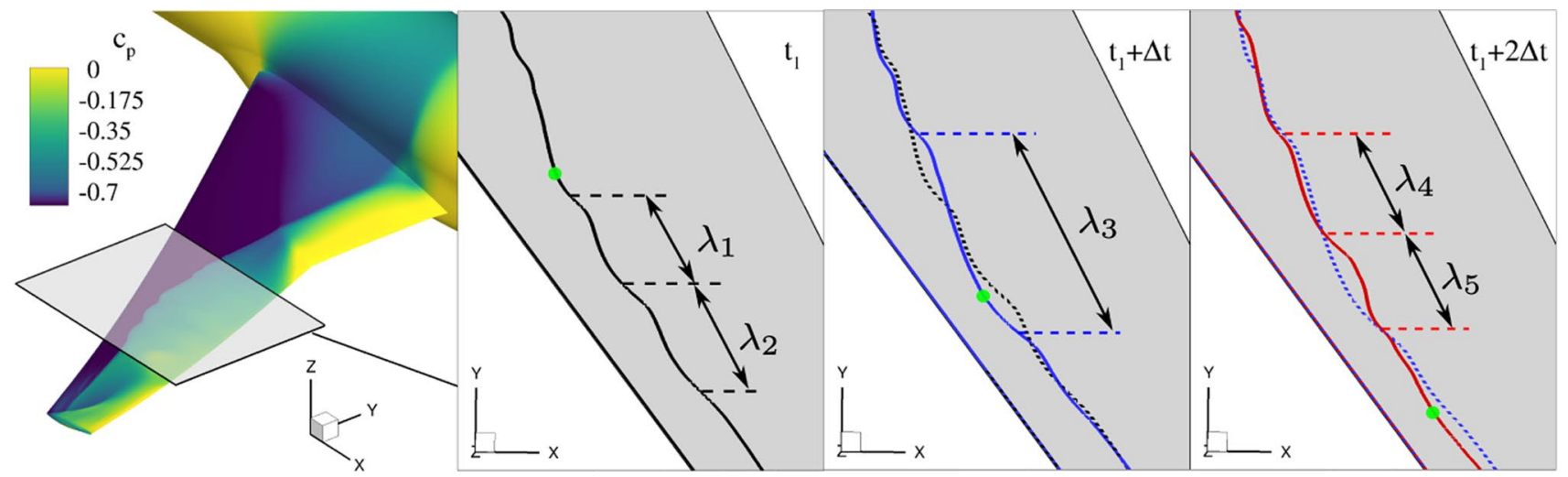

Fig. 13 Contours of instantaneous pressure coefficient for the AZDES simulation and spatial distribution and propagation of buffet cells, with time steps of $\Delta t / t_{\infty}=0.36 \times 10^{-3}$, lines of $c_{\mathrm{p}}=-0.73$, dashed line: previous snapshot, $M=0.85, \operatorname{Re}=30 \times 10^{6}, \alpha=5^{\circ}$

\subsection{Analysis of shock motion}

Figure 13 shows a series of time snapshots with $\Delta t / t_{\infty}=0.36 \times 10^{-3}$ of the area on the wing's suction side where the most distinct buffet movement can be observed. These snapshots are representative for the observed buffet behavior. The wavelengths and velocities obtained in this section are extracted from this time interval and represent a good indication for the phenomenon. On the left hand side, the instantaneous pressure coefficient of the AZDES simulation at $t_{1}$ is shown. The grey shaded square region represents the area of buffet cell motion which is focused on in the other subfigures. The first snapshot $t_{1}$ shows the shape of two buffet cells convecting in spanwise direction with wavelengths of $\lambda_{1} / \mathrm{MAC}=0.252$ and $\lambda_{2} / \mathrm{MAC}=0.296$ by means of a contour line representing $c_{\mathrm{p}}=-0.73$. A set of these periodic cells is followed by a single buffet cell with approximately twice the wavelength $\lambda_{3} / \mathrm{MAC}=0.59 \approx 2 \lambda_{1}$ in the second snapshot at $t=t_{1}+\Delta t$. The shape from the previous snapshot is indicated as a dashed line for reference. Finally, the third snapshot $t=t_{1}+2 \Delta t$ reveals a return to smaller wavelengths $\lambda_{4} / \mathrm{MAC}=0.294$ and $\lambda_{5} / \mathrm{MAC}=0.285$ and a similar flow pattern as in the first snapshot can be observed. The spanwise propagation velocity was estimated by the movement of single buffet cells, following the motion of one local spatial maximum of the pressure isoline over several time steps. The position is highlighted by a green dot in each snapshot. This was carried out for several successive buffet cells at different times in the signal, resulting in a propagation velocity that lies at $u / u_{\infty} \approx 0.24$ in the inner region and increases in the spanwise direction up to $u / u_{\infty} \approx 0.28$.

In contrast to the two-dimensional case, where a periodic shock motion characterizes transonic buffet, the observed three-dimensional buffet shows a variation in length and velocity of the buffet cells in time and in space. That observation agrees with Iovnovich et al.'s [13] findings that the buffet structure at finite swept wings is less regular than in infinite-swept cases. Timme et al. [31] observed a non-dimensional phase speed of $0.26-0.32$ on the CRM at an angle of attack of $3.75^{\circ}$ and a $R e$ number of $5 \times 10^{6}$. Despite the differences in the flow conditions, there is a fairly well agreement of the phase velocities. Even though Paladini et al. [21] investigated different wings with a 5 degrees lower sweep angle of $30^{\circ}$, the convection velocity of $(0.245 \pm 0.015) U_{\infty}$ determined in their work shows also good agreement with the values at the CRM in this investigation. The slightly higher convection speed might be due to the higher sweep angle, as indicated by Plante et al. [22]. However, the discrepancy may also be due to other geometric parameters such as taper and twist. The variations in wavelength and convection velocity also provide a possible explanation for the observed broadband frequency range connected to transonic buffet in the spectral analysis.

The spanwise extent of the buffet cells in this work, measured as the distance between crests of the $c_{\mathrm{p}}$ isoline, comprises a range between $\lambda / \mathrm{MAC}=0.25$ and 0.59 . This is lower than the wavelengths obtained by Paladini et al., which lie in a range of $\lambda / \mathrm{MAC}=0.6$ and 1.3. However, this wavelength strongly depends on the wing geometry.

\section{Conclusions}

In the present paper, URANS, DDES and AZDES simulations of transonic buffet on the CRM at flight Reynolds numbers with $M=0.85, \alpha=5^{\circ}$ and $R e=30 \times 10^{6}$ were performed. The focus was on the investigation of threedimensional transonic buffet, and the selection of a proper hybrid RANS/LES simulation approach for this complex phenomenon. Turbulence model studies showed that simulations with the SSG/LRR- $\omega$-Reynolds stress model result in a steady shock position. Therefore, the SA turbulence model with QCR extension was chosen for the presented simulations. The overall aim was to obtain reliable predictions for 
the separation location of the turbulent wake, which create a solid base for future investigations of wake turbulence. URANS and AZDES show good agreement with both the experimentally observed Strouhal numbers from wind tunnel studies and numerical predictions published in recent studies. Furthermore, a good agreement in convection velocity and the spanwise propagation of buffet cells with estimations from literature can be observed. In addition to that, a growth of the wavelength and convection velocity toward the outboard part of the wing is detected. DDES leads to a shift toward lower frequencies and does not show the characteristic spanwise convection of buffet cells. This is due to the strong interaction between the area of separated flow, which is treated in scale resolving mode in the DDES case, and the shock motion. As a consequence, AZDES is better suited for the reproduction of the experimentally observed frequency range of three-dimensional transonic buffet on the CRM than DDES.To achieve this advantage, however, AZDES requires a proper selection of the described zonal RANS/LES parameters.

Acknowledgements The authors gratefully acknowledge DLR for providing the TAU source code. Furthermore, we would like to thank the High Performance Computing Center Stuttgart (HLRS) for the support and the computational resources. The simulations were performed on the CRAY XC40 (Hazel Hen), which is funded by the Federal Ministry of Education and Research and the Ministry of Higher Education, Research and Arts Baden-Württemberg. Besides that, the work was partly funded by the German Research Association (DFG) in the project LU 809/8. This work was first presented on the 3AF Conference on separated flow in Paris, 2019.

Funding Open Access funding provided by Projekt DEAL.

Open Access This article is licensed under a Creative Commons Attribution 4.0 International License, which permits use, sharing, adaptation, distribution and reproduction in any medium or format, as long as you give appropriate credit to the original author(s) and the source, provide a link to the Creative Commons licence, and indicate if changes were made. The images or other third party material in this article are included in the article's Creative Commons licence, unless indicated otherwise in a credit line to the material. If material is not included in the article's Creative Commons licence and your intended use is not permitted by statutory regulation or exceeds the permitted use, you will need to obtain permission directly from the copyright holder. To view a copy of this licence, visit http://creativecommons.org/licenses/by/4.0/.

\section{References}

1. Ashton, N., West, A., Lardeau, S., Revell, A.: Assessment of RANS and DES methods for realistic automotive models. Comput. Fluids 128, 1-15 (2016)

2. Cecora, R.D., Radespiel, R., Eisfeld, B., Probst, A.: Differential Reynolds-stress modeling for aeronautics. AIAA J. 53(3), 736755 (2015). https://doi.org/10.2514/1.J053250

3. Crouch, J., Garbaruk, A., Magidov, D., Travin, A.: Origin of transonic buffet on aerofoils. J. Fluid Mech. 628, 357-369 (2009)
4. Crouch, J.D., Garbaruk, A., Strelets, M.: Global instability analysis of unswept-and swept-wing transonic buffet onset. In: 2018 Fluid Dynamics Conference, p. 3229 (2018)

5. Dandois, J.: Experimental study of transonic buffet phenomenon on a 3D swept wing. Phys. Fluids 28(1), 116 (2016)

6. Deck, S.: Zonal detached Eddy simulation of the flow around a high-lift configuration. AIAA J. 43(11), 2372-2384 (2005)

7. Deutsches Zentrum für Luft- und Raumfahrt: DLR TAU-Code User Guide (2018)

8. Garnier, E., Deck, S.: Large-Eddy simulation of transonic buffet over a supercritical airfoil. In: Geurts, J.F.V.A.B. (ed.) Direct and Large-Eddy Simulation VIII, pp. 549-554. Springer, Berlin (2010)

9. Giannelis, N.F., Vio, G.A., Levinski, O.: A review of recent developments in the understanding of transonic shock buffet. Prog. Aerosp. Sci. 92, 39-84 (2017)

10. Hashimoto, A., Ishida, T., Aoyama, T., Ohmichi, Y., Yamamoto, T., Hayashi, K.: Current progress in unsteady transonic buffet simulation with unstructured grid CFD code. In: 2018 AIAA Aerospace Sciences Meeting, p. 0788 (2018)

11. Schulte am Hülse, S.A.: Simulation of transonic buffet on transport aircraft using hybrid RANS/LES methods. Ph.D. thesis, German language, University of Stuttgart, Dr. Hut Verlag, Munich (2016)

12. Illi, S., Fingskes, C., Lutz, T., Krämer, E.: Transonic tail buffet simulations for the common research model. AIAA 2013-2510 (2013). https://doi.org/10.2514/6.2013-2510

13. Iovnovich, M., Raveh, D.E.: Numerical study of shock buffet on three-dimensional wings. AIAA J. 53(2), 449-463 (2014)

14. Ishida, T., Hashimoto, A., Ohmichi, Y., Aoyama, T., Takekawa, K.: Transonic buffet simulation over NASA-CRM by unsteadyFaSTAR Code. In: 55th AIAA Aerospace Sciences Meeting, p. 0494 (2017)

15. Koike, S., Ueno, M., Nakakita, K., Hashimoto, A.: Unsteady pressure measurement of transonic buffet on NASA common research model. In: 34th AIAA Applied Aerodynamics Conference, p. 4044 (2016)

16. Kok, J.C.: A high-order low-dispersion symmetry-preserving finite-volume method for compressible flow on curvilinear grids. J. Comput. Phys. 228(18), 6811-6832 (2009)

17. Lee, B.: Oscillatory shock motion caused by transonic shock boundary-layer interaction. AIAA J. 28(5), 942-944 (1990)

18. Lutz, T., Gansel, P.P.: Going for experimental and numerical unsteady wake analyses combined with wall interference assessment by using the NASA CRM-model in ETW. In: $51 \mathrm{st}$ AIAA Aerospace Sciences Meeting Including the New Horizons Forum and Aerospace Exposition, p. 871 (2013)

19. Lutz, T., Gansel, P.P., Waldmann, A., Zimmermann, D.M., Schulte am Hülse, S.A.: Time-resolved prediction and measurement of the wake past the CRM at high Reynolds number stall conditions. J. Aircr. 53(2), 501-514 (2016). https://doi. org/10.2514/1.C033351

20. Ohmichi, Y., Ishida, T., Hashimoto, A.: Modal decomposition analysis of three-dimensional transonic buffet phenomenon on a swept wing. AIAA J. 56(10), 3938-3950 (2018)

21. Paladini, E., Dandois, J., Sipp, D., Robinet, J.C.: Analysis and comparison of transonic buffet phenomenon over several threedimensional wings. AIAA J. (2018). https://doi.org/10.2514/1. J056473

22. Plante, F., Dandois, J., Sartor, F., Laurendeau, E.: Study of three-dimensional transonic buffet on swept wings. In: 35th AIAA Applied Aerodynamics Conference p. 3903 (2017)

23. Sartor, F., Timme, S.: Delayed detached Eddy simulation of shock buffet on half wing-body configuration. AIAA J. 55(4), 1230-1240 (2016) 
24. Schwamborn, D., Gerhold, T., Heinrich, R.: The DLR TAUcode, recent applications in research and industry. In: European Conference on Computational Fluid Dynamics ECCOMAS CFD 2006, (2006)

25. Spalart, P.R.: Strategies for turbulence modelling and simulations. Int. J. Heat Fluid Flow 21(3), 252-263 (2000)

26. Spalart, P.R., Allmaras, S.R.: A one-equation turbulence model for aerodynamic flows. Recherche Aerospatiale 1, 5-21 (1994)

27. Spalart, P.R., Deck, S., Shur, M.L., Squires, K.D., Strelets, M., Travin, A.: A new version of detached-Eddy simulation, resistant to ambiguous grid densities. Theoret. Comput. Fluid Dyn. 20(3), 181 (2006)

28. Spalart, P.R., Streett, C.: Young-person's guide to detachededdy simulation grids. Technical report, NASA CR-2001$211032(2001)$

29. Sugioka, Y., Koike, S., Nakakita, K., Numata, D., Nonomura, T., Asai, K.: Experimental analysis of transonic buffet on a $3 \mathrm{~d}$ swept wing using fast-response pressure-sensitive paint. Exp. Fluids 59(6), 108 (2018). https://doi.org/10.1007/s0034 8-018-2565-5

30. Thiery, M., Coustols, E.: Numerical prediction of shock induced oscillations over a 2D airfoil: influence of turbulence modelling and test section walls. Int. J. Heat Fluid Flow 27, 661-670 (2006)
31. Timme, S.: Global Instability of Wing Shock-Buffet Onset. J. Fluid Mech. 885, A37 (2020). https://doi.org/10.1017/jfm.2019.1001

32. Togiti, V., Eisfeld, B., Brodersen, O.: Turbulence model study for the flow around the NASA common research model. J. Aircr. 51(4), 1331-1343 (2014)

33. Vassberg, J.C., DeHaan, M.A., Rivers, M.B., Wahls, R.A.: Development of a common research model for applied CFD validation studies. In: 26th AIAA Applied Aerodynamics Conference (2008). https://doi.org/10.2514/6.2008-6919

34. Waldmann, A., Gansel, P.P., Lutz, T., Krämer, E.: Unsteady wake of the NASA common research model in low-speed stall. J. Aircr. 53(4), 1073-1086 (2016). https://doi.org/10.2514/1.C033413

Publisher's Note Springer Nature remains neutral with regard to jurisdictional claims in published maps and institutional affiliations. 